\title{
Exercise Counseling to Enhance Smoking Cessation Outcomes: The Fit2Quit Randomized Controlled Trial
}

\author{
Ralph Maddison, PhD • Vaughan Roberts, PhD • Hayden McRobbie, PhD • \\ Christopher Bullen, PhD • Harry Prapavessis, PhD • Marewa Glover, PhD • \\ Yannan Jiang, PhD • Paul Brown, PhD • William Leung, MSc • Sue Taylor • Midi Tsai, MSc
}

Published online: 7 March 2014

(C) The Society of Behavioral Medicine 2014

\begin{abstract}
Background Regular exercise has been proposed as a potential smoking cessation aid.

Purpose This study aimed to determine the effects of an exercise counseling program on cigarette smoking abstinence at 24 weeks.

Methods A parallel, two-arm, randomized controlled trial was conducted. Adult cigarette smokers $(n=906)$ who were insufficiently active and interested in quitting were randomized to receive the Fit2Quit intervention (10 exercise telephone counseling sessions over 6 months) plus usual care
\end{abstract}

R. Maddison $(\bowtie) \cdot$ V. Roberts $\cdot$ C. Bullen $\cdot$ Y. Jiang $\cdot$ M. Tsai National Institute for Health Innovation, University of Auckland,

Private Bag 92019, Auckland 1142, New Zealand

e-mail: r.maddison@nihi.auckland.ac.nz

H. McRobbie

UK Centre for Tobacco Control Studies, Wolfson Institute of Preventive Medicine, Barts \& The London School of Medicine and

Dentistry, Queen Mary, University of London, London, UK

H. Prapavessis

School of Kinesiology, Faculty of Medical and Health Sciences, University of Western Ontario, 1151 Richmond St,

London, ON, Canada N6A 3K7

M. Glover

Centre for Tobacco Control Research, Social and Community Health, School of Population Health, University of Auckland, Private Bag 92019, Auckland 1142, New Zealand

\section{P. Brown}

School of Social Sciences, Humanities, and Arts, University of California, Merced, CA, USA

W. Leung

Epidemiology and Biostatistics, University of Auckland,

AucklandPrivate Bag 92019, New Zealand

S. Taylor

T \& T Consulting, PO Box 311, Levin, New Zealand (behavioral counseling and nicotine replacement therapy) or usual care alone.

Results There were no significant group differences in 7-day point-prevalence and continuous abstinence at 6 months. The more intervention calls successfully delivered, the lower the probability of smoking (OR, 0.88 ; $95 \%$ CI $0.81-0.97, p=0.01$ ) in the intervention group. A significant difference was observed for leisure time physical activity (difference $=219.11$ METminutes/week; $95 \%$ CI 52.65-385.58; $p=0.01$ ).

Conclusions Telephone-delivered exercise counseling may not be sufficient to improve smoking abstinence rates over and above existing smoking cessation services. (Australasian Clinical Trials Registry Number: ACTRN12609000637246.)

Keywords Exercise · Addiction · Smoking cessation · Behavior change $\cdot$ Randomized controlled trial

\section{Introduction}

The adverse health effects of tobacco smoking are well described including a wide range of cancers, cardiovascular disease, chronic obstructive pulmonary disease, other chronic diseases, and pregnancy complications [1]. Tobacco smoking is the world's leading preventable cause of death [2], with nearly six million deaths each year attributable to tobacco use. Despite the availability in many countries of effective treatments for smoking dependence (such as behavioral support and pharmacotherapies), the proportion of people who successfully quit smoking and remain abstinent is disappointingly low [3-5]. New approaches to support the implementation of existing evidence-based smoking cessation treatments are needed. One proposed additional aid is exercise [6].

Smoking cessation is associated with withdrawal symptoms such as sleep disturbance, irritability, and craving and weight gain [7]. Many of these negative effects, including 
symptoms of anxiety and psychosocial stress, have been shown to be ameliorated by exercise [8]. Two recently published meta-analyses suggest that acute bouts of moderate exercise significantly reduce the magnitude of urges to smoke compared with control conditions $[9,10]$. As a consequence, there has been increased attention in recent years regarding the role of exercise as a smoking cessation intervention [6].

A Cochrane review [6] of exercise interventions for smoking cessation identified 15 randomized controlled trials $(n=4298)$ with adequate follow-up (i.e., at least 6 months after the quit date). All but one of these studies examined the effect of exercise in combination with standard smoking cessation treatment and compared outcomes with a control group who also received smoking cessation treatment. Only 3 [11-13] of the 15 trials showed significantly higher abstinence rates in a physically active group compared with a control group at the end of treatment, and only one showed a significant benefit of exercise at follow-up [12].

Methodological issues associated with these trials have limited the ability to reliably measure the effect of exercise on smoking cessation rates: Seven trials in the Cochrane review had fewer than 25 participants in each treatment arm, and lacked statistical power to detect an effect; six trials recruited only women, and one recruited only men, limiting the generalizability of the findings; the exercise interventions may not have been sufficiently intense or of sufficient duration to have had an effect on smoking outcomes; finally, the majority of studies to date can be classified as efficacy trials and have not been conducted in "real world" settings to augment existing effective treatments. We sought to address these methodological concerns and to determine whether integrating two existing national programs, Quitline and Green Prescription, both proven effective for smoking cessation [14, 15] and physical activity promotion [16], respectively, could enhance abstinence rates compared to Quitline support alone.

\section{Methods/Design}

Full details of the design and conduct of the Fit2Quit trial have been published elsewhere [17]. In brief, a parallel two-arm randomized controlled trial was conducted in New Zealand between 2009 and 2012. Eligible participants were randomized at 1:1 ratio to either receive an exercise intervention plus usual stop smoking support, or usual stop smoking support alone (i.e., usual care). The randomization sequence was generated by the study biostatistician and was stratified by study center (Auckland and Waikato), sex, and ethnicity (Māori [indigenous] and non-Māori). Participants were enrolled by trained study research assistants and assigned to interventions. Concealment of allocation was ensured by means of a central computerized service up to the point of randomization. The study was registered with the Australasian Clinical Trials Registry (ACTRN12609000637246).

\section{Participants and Recruitment}

Study procedures and terms were approved by the New Zealand Multi-region Ethics Committee (MEC/09/08/090). Participants were recruited from the Auckland and Waikato regions (joint population of 2.0 million) when they called Quitline, a national smoking cessation telephone support program. Participants were eligible if they were daily smokers, at least 18 years of age, self-reported being interested in quitting and indicated that intention by having already phoned Quitline, wanted to be physically active, smoked their first cigarette within $30 \mathrm{~min}$ of waking, and could provide informed consent. Participants were excluded if they had had a stroke or heart related condition in the previous 2 weeks, were enrolled in a competing smoking cessation program, had a medical condition that limited their ability to exercise safely, were already participating in an exercise program, or were already meeting the recommended 150 min of physical activity per week. Recruitment began in January 2010 and ended in August 2011.

\section{Procedure and Setting}

During the registration phone call, eligible participants were offered either a telephone or face-to-face assessment: Both assessments collected identical information. Research assistants conducted all assessments according to standard procedures. Participants completed questionnaires on smoking history, current smoking status, and physical activity at the baseline assessment. Participants were randomized at the end of the assessment to either intervention or control groups. Repeat assessments were conducted at 8 and 24 weeks after the nominated quit date. Study researchers conducting these assessments were not blinded to treatment allocation. The final 24-week assessment was completed in February 2012.

\section{Measures}

Demographics and smoking history, including the Fagerström Test of Nicotine Dependence [18], were collected at baseline. Tobacco withdrawal symptoms were assessed with the Mood and Physical Symptoms Scale [19]. Physical activity levels were assessed using the International Physical Activity Questionnaire long form (IPAQ, 20). The International Physical Activity Questionnaire was developed to obtain international and comparable estimates of physical activity and the long version was used in this study because it is suitable for research or evaluation purposes. The sensitivity of selfreported instruments to change is difficult to assess and the metrics for this are often poorly described, but the 
International Physical Activity Questionnaire is an appropriate measure and provides important contextual information on activity domains, including at work (physical activity undertaken as part of one's job), at home (physical activity undertaken during domestic duties or gardening), in active transport (physical activity undertaken while commuting), and during leisure time (physical activity undertaken solely for exercise, sport, or recreation). In accordance with International Physical Activity Questionnaire guidelines [20], results are reported in metabolic equivalent (MET)-minutes/week.

\section{Usual Care Smoking Cessation}

All enrolled participants received usual smoking cessation services delivered by smoking cessation advisors at Quitline [15], who provide information and support to quit smoking. Participants were encouraged to set a quit date and offered one-to-one telephone support for 3 months, as well as an exchange card (Quitcard) for up to 8 weeks of subsidized nicotine replacement therapy (patches, gum, or lozenge) to be redeemed at a local community pharmacy. The control group was assigned to usual care alone.

\section{Intervention}

Participants randomized to the intervention group received a comprehensive 6-month home and community-based exercise program delivered by Green Prescription [21] services in addition to usual Quitline smoking cessation support. The Green Prescription exercise intervention was chosen as it has been shown to be effective at increasing physical activity among inactive adults [16]. Green Prescription involves a referral from primary care to agencies that support physical activity [16]. In this trial, however, registered participants randomized to the intervention group were referred by the researcher, without involving the General Practitioner. Once referred, trained exercise facilitators (patient support persons) contacted participants and offered telephone counseling to promote and support exercise behavior. The original program involves monthly contacts for a total of 3 months; however, given the increased response burden produced by trying to change two behaviors at once [22], the number of contacts was increased to maximize its impact. A total of 10 contacts (face-to-face and telephone support sessions) were delivered over 6 months, with the goal of having individuals participating in a minimum of $30 \mathrm{~min}$ of moderate-vigorous aerobicbased exercise on most days of the week, in line with current recommendations [23].

The intervention was developed from Social Cognitive Theory [24] and aimed to enhance participant's efficacious beliefs to be physically active. Self-efficacy refers to one's situational confidence to perform a specific task or behavior and has been shown to facilitate exercise initiation and maintenance in adults [25]. Moreover, self-efficacy based interventions have proven effective at increasing physical activity behavior and adherence [26, 27]. Patient support persons were provided with comprehensive training on selfefficacy and motivation constructs, as well as telephone counseling with motivation interviewing concepts and techniques [17] by the research team, in accordance with the International Conference on Harmonization Good Clinical Practice guidelines. Full details of the intervention are provided elsewhere [17]; however, Table 1 summarizes the content of each of the intervention contacts.

All intervention telephone calls, including those with successful or unsuccessful contact, were logged to evaluate adherence with the intervention. For logistic reasons (cost, returning devices), it was not possible to objectively measure exercise adherence with a movement device (e.g., accelerometers). Intervention phone calls were also monitored throughout the study to ensure the fidelity of the intervention delivery. To achieve this, each patient support person conducted a faceto-face interview and a "phone call 6" (See Table 1) in the presence of two study researchers, who provided feedback focusing on any aspects of the intervention that were missed. This process was repeated every 6 months and when a new patient support person was employed.

\section{Outcomes}

The primary outcome was self-reported point-prevalence (i.e., not a single puff of a cigarette in the past 7 days) at 24 weeks after the nominated quit date. Verification of quit status was not undertaken due to the use of telephone-based assessments. Previous reviews of smoking cessation studies have shown that rates of misreporting of smoking abstinence are generally $<5 \%$ [28]. Limited face-to-face contact time has been identified as a circumstance under which the added benefits of biochemical verification of abstinence are outweighed by the burden (or cost) of obtaining such information [29]. The secondary outcomes were self-reported continuous abstinence (i.e., no more than five cigarettes since the quit date) at 24 weeks after the nominated quit date, self-reported 7-day point-prevalence at 8 weeks, change from baseline in tobacco withdrawal symptoms, self-rated chances of quitting, and selfreported physical activity levels measured with the International Physical Activity Questionnaire.

\section{Sample Size}

An a priori sample size estimate indicated that 1,400 participants (700 per arm) were required to provide $90 \%$ power at $5 \%$ level of significance to detect an increase in quit rates over baseline from 15 to $22.5 \%$ at 6 months, assuming a dropout rate of $20 \%$. A $7.5 \%$ difference between groups represents a 
Table 1 Summary of the content of each intervention contact

\begin{tabular}{|c|c|c|c|}
\hline Contact & Time & Session content & Self-efficacy variable \\
\hline \multirow[t]{7}{*}{ 1-face-to-face } & Week 1 & Welcome participant to the programme & \\
\hline & & Explain the positive effects of regular physical activity on physical and mental heath & Outcome expectancy \\
\hline & & $\begin{array}{l}\text { Obtain exercise history-what activities have you enjoyed participating in the past? } \\
\text { What time of day do you prefer to exercise? What activities do you currently enjoy? } \\
\text { In the past what factors helped you to participate in regular physical activity, and } \\
\text { what factors hindered you? }\end{array}$ & Mastery experiences \\
\hline & & $\begin{array}{l}\text { Discuss normal physiological responses to exercise - highlighting what they are likely } \\
\text { to experience }\end{array}$ & Physiological responses \\
\hline & & $\begin{array}{l}\text { Explain the benefit of monitoring progress with a pedometer and exercise log. Explain } \\
\text { perceived exertion scale to monitor intensity of exercise }\end{array}$ & $\begin{array}{l}\text { Mastery experiences and } \\
\text { task efficacy }\end{array}$ \\
\hline & & Exercise prescription - prescribe exercise based on exercise history & Task efficacy \\
\hline & & Social persuasion — suggest the possibility of exercising with friends/family & $\begin{array}{l}\text { Social persuasion/peer } \\
\text { modeling }\end{array}$ \\
\hline
\end{tabular}

Discuss relapse - and the fact that if "slips" occur (i.e., the participant has a cigarette, or misses an exercise session) they should not give up

2 - phone call 1 Week 2 Discuss the barriers to exercise faced in the last week

Discuss the physiological responses to exercise experienced in the previous week. Based on responses, reinforce that these are normal responses to exercise and any discomfort often improves with time

Discuss goal setting using the SMART principle — set specific, measurable, action-oriented, realistic, time-based goals

3 - phone call 2 Week 3 Discuss common barriers to exercise - use the IDEA approach - identify barriers, develop a strategy, evaluate, assess. Prioritize the most common barriers to exercise and develop a strategy to overcome them

4 -phone call 3 Week 4 Discuss the approach of changing the participant's perspective to exercise, from one of fitting exercise into their life to fitting their life around exercise

Work through another barrier using the IDEA approach

Identify high risk situations that will challenge the participant's exercise compliance coping skills. Encourage the participant to seek out situations that make exercise convenient and enjoyable, and to avoid people, places, or situations that may affect their ability to adhere to their exercise program

5-phone call 4 Week 6 Draw the participants attention to where they started and how far they have come

Discuss how physiological responses to exercise have improved over time

6- phone call 5 Week 8 Plan long-term maintenance of exercise behavior by discussing opportunities for physical activity at local community facilities and organisations

7-phone call 6 Week 12 Discuss how to maintain focus on exercise in different environments, such as when on holiday — attempt to maintain a similar routine, use exercise as a way to explore surroundings, etc.

8 - phone call 7 Week 16 Discuss community options for exercise. Suggest exercise options in the community based on the participant's exercise interests. Set some goals regarding community exercise (if appropriate)

9-phone call 8 Week 20 Discuss community options for exercise. Ask the participant what activities they will continue to do in the community environment. Discuss location, cost, transport, with the participant. Help facilitate the participant joining an activity program if needed

10 - phone call 9 Week 24 Review previous 6 months, reinforcing progress made to date, fitness improvements, barriers overcome, etc.

Ensure participant is aware they need to continue to exercise regularly, maintaining or exceeding their prescribed level of exercise, scheduling exercise into their day, and exercising with others and/or participating in exercise options in the community (if appropriate)

All calls Review progress since last contact. Provide positive feedback and encouragement regarding progress

Discuss pedometer counts and exercise log

Barrier efficacy

Physiological responses

Barrier efficacy

Scheduling efficacy

Barrier efficacy

Coping efficacy

Mastery experiences

Physiological responses

Coping efficacy

Coping efficacy

Barrier and scheduling efficacy

Mastery experiences

Provide positive affirmations, support, and encouragement regarding the participants progress Social persuasion

Discuss relapse if required. If relapse has occurred, discuss "coping self-talk" with the participant, such as "I'll continue to exercise as I did before...I know that once I get started, I really enjoy exercising," etc.

Set goals for the time period until the next contact 
$50 \%$ improvement on quit rates, which was considered realistic, yet clinically significant.

There were difficulties with recruitment early in the trial, and we calculated that a final sample of 970 participants (485 per group) would provide $90 \%$ power at $5 \%$ level of significance to detect a minimum difference of $10 \%$ in smoking cessation rates, adjusting for $20 \%$ loss to follow up.

\section{Statistical Analysis}

Statistical analyses were conducted in 2012 using SAS version 9.2 (SAS Institute Inc., Cary, NC, USA) and R version 2.14.1 (R Foundations for Statistical Computing). All statistical tests were two-tailed, and a $5 \%$ significance level was maintained throughout the analyses. Treatment evaluations were performed on the principle of intention-to-treat using all the data collected from randomized participants. Those participants whose smoking status could not be ascertained at follow-up were assumed to be smoking, as recommended for smoking cessation studies [30].

Chi-squared analyses were used to evaluate the main treatment effect on the primary outcome, with estimation of relative risks ( $95 \%$ confidence intervals). As a secondary analysis, the effect of the intervention was evaluated using a logistic regression model adjusting for baseline stratification factors, age in years, and strength of urge to smoke. Odds ratios (ORs), $95 \%$ confidence intervals, and two-sided $p$ values are presented for comparison. Statistical tests and regression models appropriate for continuous and categorical data were used for secondary outcomes.

\section{Results}

Figure 1 shows participant flow. A total of 906 trial participants were randomized, with 455 and 451 in the intervention and control groups, respectively. For intervention and control groups, loss-to-follow-up rates were 7 and $1 \%$, respectively, at 8 weeks and increased to 11 and $4 \%$, respectively, at 24 weeks.

Table 2 shows the baseline characteristics of all randomized participants, with no observed differences between intervention and control groups. The mean overall score for the Fagerström Test of Nicotine Dependence was 5.6, indicating a moderate level of dependence (data not shown).

While all participants set a quit date, only 75 and $69 \%$ of control and intervention group participants, respectively, actually quit (17 and $24 \%$ of these data were missing).

Cessation Outcomes Smoking abstinence rates at 24 weeks were moderately high but not significantly different between intervention and control groups. For 7-day point prevalence, the abstinence rates were $23 \%$ in the intervention group (105 out of 455 ) and $22 \%$ in the control group (98 out of 451$)$. The relative risk (RR) for smoking was 0.98 (95\% CI 0.92-1.05; $p=0.63$ ). Continuous abstinence rates were $17 \%$ (78 out of 455 ) and $18 \%$ (80 out of 451) in intervention and control groups, respectively; RR for smoking was 1.01 (95\% CI $0.95-1.07 ; p=0.81$ ). The ORs estimated in adjusted logistic regression analyses were close to the RRs with no significant treatment differences between groups. For those baseline factors adjusted in the regression model, the probability of smoking was significantly higher in Māori participants (OR $1.63 ; 95 \%$ CI 1.12-2.38, $p=0.01)$ and those with higher baseline strength of urge to smoke (OR 1.28; $95 \%$ CI 1.11$1.47, p=0.0006$ ) in 7-day point prevalence. Similar ORs were found in continuous abstinence (data not shown).

Sensitivity Analysis No significant differences were found between groups for 7-day point prevalence or continuous abstinence using observed data only (i.e., no imputation). For 7-day point prevalence, the total number of complete cases was 683 ( $75 \%$ of those randomized, $N=906)$, with 328 and 355 in the intervention and control groups, respectively. Of these, 105 participants in the intervention group and 98 in the control group self-reported quitting at 24 weeks (adjusted OR for smoking, 0.86; $95 \%$ CI 0.62-1.21, $p=$ 0.39 ). For continuous abstinence, the total number of complete cases was $680(75 \%)$ with 326 and 354 in the intervention and control groups, respectively. Of these, 78 participants in the intervention group and 80 in the control group reported quitting at 24 weeks (adjusted OR for smoking 1.01; $95 \% \mathrm{CI}$ $0.70-1.45, p=0.97$ ) (data not shown).

Adherence to the Intervention and Cessation Outcomes Of the 455 participants who were randomized to the intervention group, 236 (52\%) completed at least seven of the 10 intervention calls (median $=7$, interquartile range $=4-9$ ). One hundred twenty-five participants received less than half of the scheduled intervention calls ( $\leq 4$ calls). The number of intervention calls delivered significantly reduced the probability of smoking at 24 weeks (OR, $0.88 ; 95 \%$ CI $0.81-0.97, p=0.01)$ for those in the intervention group. Moreover, when compared with the control group, a significant treatment effect on smoking cessation at 24 weeks was found for those who received seven intervention calls or more (OR, 0.67; $95 \%$ CI $0.46-0.98, p=0.04)$.

Smoking Withdrawal and Behavior There were no statistically significant intervention effects on tobacco withdrawal symptoms. For total mood and physical symptoms scores (range, 1-25), the model adjusted means were 9.90 (SE, 0.21 ) and 9.92 (SE, 0.20) in the intervention and control groups, respectively $(p=0.96)$. However, there was a small but statistically significant difference in the number of cigarettes smoked in the previous 7 days (mean difference, -0.92 
Fig. 1 Participant flow

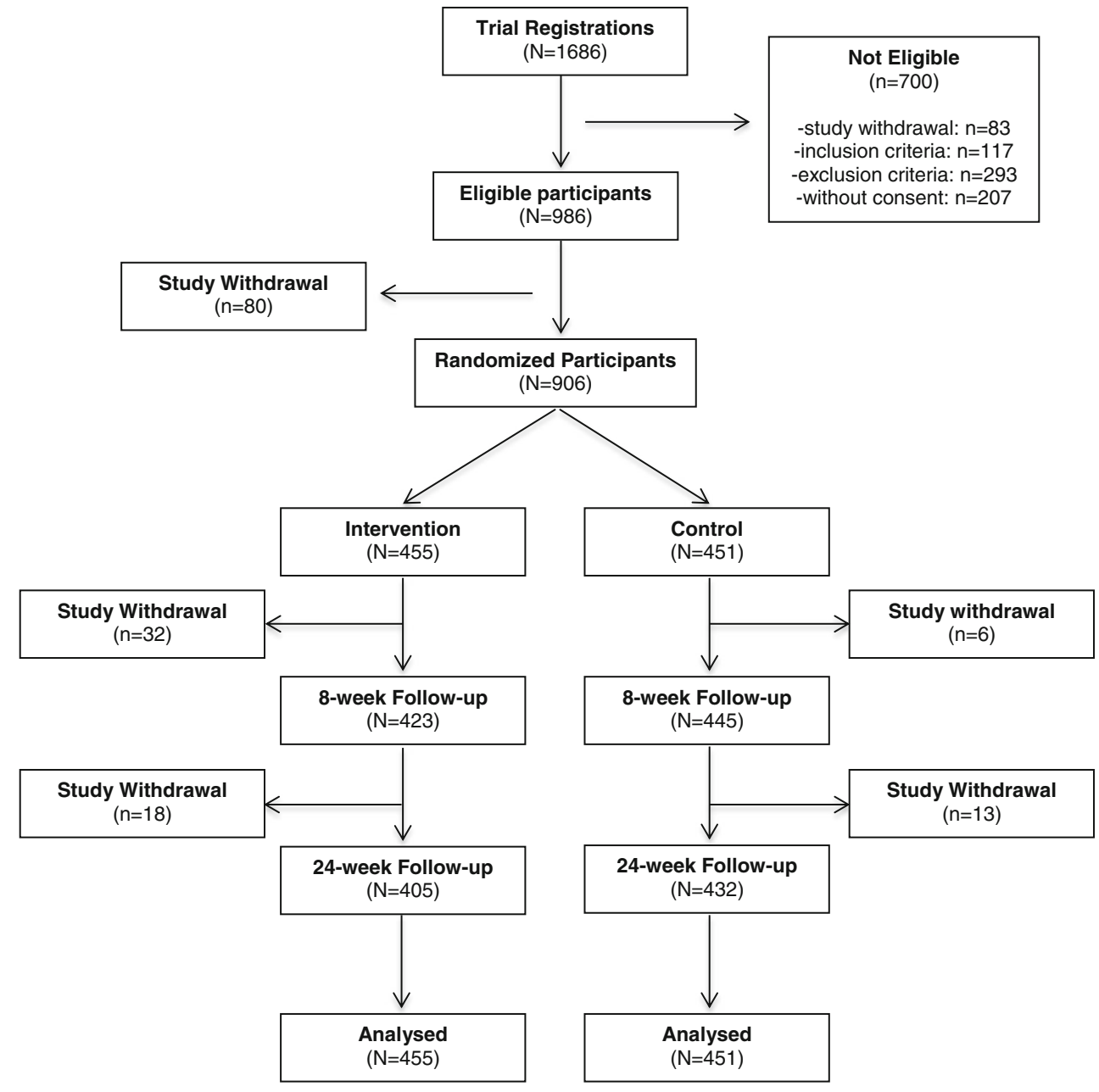

cigarettes per day; $95 \%$ CI $0.06-0.39 ; p=0.006$ ) as well as in the number of cigarettes smoked since participants' nominated quit date (mean difference, -1.01 cigarettes per day; $95 \%$ $\mathrm{CI}-1.74$ to $-0.09, p=0.02$ ) in favor of the intervention group. Self-rated chance of quitting was also significantly lower in the intervention group compared to those in the control at 8 weeks (mean difference, $0.23 ; 95 \% \mathrm{CI}-1.90$ to -0.12 ; $p=$ $0.02)$, see Table 3.

Nicotine Replacement Therapy Use At baseline, 200 (44\%) and 205 (45\%) participants were using nicotine replacement therapy in the control and intervention groups, respectively, while another 202 and 195, respectively, indicated that they had "not got around to it" or the nicotine replacement therapy products had not arrived yet. There was no effect of baseline nicotine replacement therapy use on 7-day point prevalence abstinence at 24 weeks (OR, 1.10; $95 \%$ CI 0.67, 1.83, $p=$ $0.70)$ in the regression model.

Physical Activity Outcomes A positive intervention effect was found for leisure time physical activity. Overall, an increase of 526 (SE, 69.18) MET minutes per week was observed in the intervention group and 307 (SE 66.11) observed in the control group at 24 weeks (difference, 219.11; 95 \% CI 52.65-385.58, $p=0.01$; see Table 4 ). There were no other significant differences between groups for the other activity domains.

Adverse Events A total of 15 (3\%) and 14 (3\%) participants reported serious adverse events in the control and intervention groups, respectively, but none were deemed related to the intervention.

\section{Discussion}

The major findings of the present study can be summarized as follows. In line with many previous studies, no differences in smoking abstinence rates between the exercise intervention and control conditions were found; however, there was a very small effect on the number of cigarettes smoked in the previous week, which favored the intervention group. There was no intervention effect for tobacco withdrawal symptoms. Taken together, these findings suggest that telephone delivered exercise counseling may not be sufficient to improve cessation 
Table 2 Baseline characteristics for all randomized participants

\begin{tabular}{|c|c|c|c|}
\hline & Control $(n=451)$ & Intervention $(n=455)$ & Total $(n=906)$ \\
\hline Age (years), mean $\pm \mathrm{SD}$ & $37.3 \pm 12.2$ & $37.6 \pm 12.2$ & $37.5 \pm 12.2$ \\
\hline \multicolumn{4}{|l|}{ Sex } \\
\hline Male, $n(\%)$ & $207(45.9)$ & $208(45.7)$ & $415(45.8)$ \\
\hline Female, $n(\%)$ & $244(54.1)$ & $247(54.3)$ & $491(54.2)$ \\
\hline \multicolumn{4}{|l|}{ Prioritised ethnicity } \\
\hline Māori, $n(\%)$ & $138(30.6)$ & $142(31.2)$ & $280(30.9)$ \\
\hline Pacific, $n(\%)$ & $55(12.2)$ & $47(10.3)$ & $102(11.3)$ \\
\hline NZ European, $n(\%)$ & $214(47.5)$ & $218(47.9)$ & $432(47.7)$ \\
\hline Asian, $n(\%)$ & $11(2.4)$ & $13(2.9)$ & $24(2.7)$ \\
\hline Other, $n(\%)$ & $33(7.3)$ & $35(7.7)$ & $68(7.5)$ \\
\hline Number of cigarettes/day, mean \pm SD & $19.83 \pm 9.2$ & $19.41 \pm 9.5$ & $19.62 \pm 9.3$ \\
\hline Age of smoking onset, mean \pm SD & $15.47 \pm 4.2$ & $15.54 \pm 4.2$ & $15.5 \pm 4.2$ \\
\hline Years smoking continuously, mean $\pm \mathrm{SD}$ & $20.48 \pm 11.9$ & $20.15 \pm 12.1$ & $20.22 \pm 12.0$ \\
\hline Fagerström Test of Nicotine Dependence score, mean \pm SD & $5.63 \pm 1.95$ & $5.52 \pm 1.90$ & $5.57 \pm 1.93$ \\
\hline \multicolumn{4}{|l|}{ Previous quit attempts } \\
\hline Yes, $n(\%)$ & $348(77.2)$ & $368(80.9)$ & $716(79)$ \\
\hline No, $n(\%)$ & $103(22.8)$ & $87(19.1)$ & $190(21)$ \\
\hline \multicolumn{4}{|l|}{ Number of quit attempts in previous 12 months } \\
\hline One, $n(\%)$ & $83(23.9)$ & $93(25.3)$ & $176(24.6)$ \\
\hline Two, $n(\%)$ & $28(8.1)$ & $37(10.1)$ & $65(9.1)$ \\
\hline Three, $n(\%)$ & $14(4.0)$ & $15(4.1)$ & $29(4.1)$ \\
\hline Four or more, $n(\%)$ & $18(5.2)$ & $20(5.4)$ & $38(5.3)$ \\
\hline None, $n(\%)$ & $204(58.6)$ & $202(54.9)$ & $406(56.7)$ \\
\hline Do not know, $n(\%)$ & $1(0.3)$ & $1(0.3)$ & $2(0.3)$ \\
\hline \multicolumn{4}{|l|}{ Confidence to quit } \\
\hline One (very low), $n(\%)$ & $8(1.8)$ & $7(1.5)$ & $15(1.7)$ \\
\hline Two, $n(\%)$ & $15(3.3)$ & $12(2.6)$ & $27(3.0)$ \\
\hline Three, $n(\%)$ & $97(21.5)$ & $83(18.2)$ & $180(19.9)$ \\
\hline Four, $n(\%)$ & $140(31.0)$ & $148(32.5)$ & $288(31.8)$ \\
\hline Five (very high), $n(\%)$ & $190(42.1)$ & $204(44.8)$ & $394(43.5)$ \\
\hline Missing, $n(\%)$ & $1(0.2)$ & $1(0.2)$ & $2(0.2)$ \\
\hline \multicolumn{4}{|l|}{ Physical activity (MET minutes/week), mean \pm SD } \\
\hline Leisure time domain & $601 \pm 1,150$ & $557 \pm 1,094$ & $579 \pm 1,122$ \\
\hline Work domain & $3,557 \pm 6,370$ & $3,998 \pm 6,800$ & $3,777 \pm 6,589$ \\
\hline Active transport domain & $437 \pm 1,047$ & $430 \pm 1403$ & $433 \pm 1,238$ \\
\hline Domestic and garden domain & $1,838 \pm 2,877$ & $1,792 \pm 2,640$ & $1,815 \pm 2,759$ \\
\hline Total walking & $1,769 \pm 2,838$ & $1,744 \pm 2,624$ & $1,756 \pm 2,731$ \\
\hline Total moderate physical activity & $3,191 \pm 3,728$ & $3,316 \pm 3,976$ & $3,254 \pm 3,853$ \\
\hline Total vigorous physical activity & $1,472 \pm 3,728$ & $1,733 \pm 4,155$ & $1,602 \pm 3,947$ \\
\hline
\end{tabular}

rates over and above existing smoking cessation services such as Quitline. However, treatment adherence was associated with reduced probability of smoking among the intervention group so the study findings may have been different if intervention adherence had been higher.

Despite the null effect observed on smoking abstinence, this study extends the literature in this area because it is the first to leverage existing national delivery services for both smoking cessation and physical activity promotion. The Green Prescription program was chosen because it has been shown to be effective at increasing levels of physical activity among inactive adults [16] and was effective at increasing leisure time physical activity in the present study, the domain targeted in the intervention, rather than occupational activity. We focused on domain-specific physical activity (leisure time), which is consistent with recommendations by 
Table 3 Tobacco withdrawal symptoms and smoking behavior outcomes

\begin{tabular}{|c|c|c|c|c|c|c|c|}
\hline \multirow[t]{2}{*}{ Study outcomes } & \multirow{2}{*}{$\begin{array}{l}\text { Intervention } \\
\text { Estimate (SE) }\end{array}$} & \multirow{2}{*}{$\begin{array}{l}\text { Control } \\
\text { Estimate } \\
\text { (SE) }\end{array}$} & \multicolumn{5}{|c|}{ Estimated effect size (intervention-control) } \\
\hline & & & $\begin{array}{l}\text { Difference } \\
\text { in means }\end{array}$ & $\mathrm{SE}$ & $\begin{array}{l}95 \% \text { lower } \\
\text { CI }\end{array}$ & $\begin{array}{l}95 \% \text { upper } \\
\text { CI }\end{array}$ & $P$ value \\
\hline Total MPSS ${ }^{\mathrm{a}}$ score $(1-25)^{\mathrm{b}}$ & $9.90(0.21)$ & $9.92(0.20)$ & -0.01 & 0.25 & -0.51 & 0.49 & 0.9601 \\
\hline Total MPSS $^{\mathrm{a}}$ score (self-reported abstainers only) ${ }^{\mathrm{b}}$ & $8.57(0.31)$ & $8.23(0.32)$ & 0.35 & 0.39 & -0.41 & 1.11 & 0.3691 \\
\hline Number of cigarettes smoked per day in the past 7 days $^{b}$ & $4.75(0.34)$ & $5.67(0.34)$ & -0.92 & 0.42 & -1.74 & -0.09 & 0.0296 \\
\hline Number of cigarettes smoked per day since nominated quit date $\mathrm{b}^{\mathrm{b}}$ & $5.07(0.37)$ & $6.08(0.36)$ & -1.01 & 0.45 & -1.90 & -0.12 & 0.0255 \\
\hline Self-rated chance of quitting at 8 weeks ${ }^{\mathrm{c}}$ & $3.96(0.07)$ & $3.73(0.07)$ & 0.23 & 0.08 & 0.06 & 0.39 & 0.0067 \\
\hline
\end{tabular}

${ }^{\text {a }}$ Mood and physical symptoms scale

${ }^{\mathrm{b}}$ Repeated measures mixed model adjusted for: baseline outcome (as appropriate), age, sex, Māori, center, baseline strength of urge to smoke, and time

${ }^{\mathrm{c}}$ ANCOVA Model adjusted for: baseline outcome, age, sex, Māori, center, and baseline strength of urge to smoke

developers of the International Physical Activity Questionnaire [31]. There were no statistically significant effects of the intervention on the other nontargeted domains; however, descriptive data suggest greater increases for the intervention in the work domain, active transport, and vigorous exercise, but not for domestic activities or moderate intensity. Collectively, these data suggest a reallocation of time spent on physical activities.

Measurement issues also warrant discussion. While the International Physical Activity Questionnaire-Long Form is appropriate for research purposes, provides domain specific information, and permits international comparison, its responsiveness to change is less clear [31]. In addition, there are concerns regarding the interpretation of the high prevalence estimates obtained from this measure [31]. Future research should incorporate more objective measures of physical activity and intensity, such as accelerometry.

The effects of the intervention on physical activity should be interpreted with caution because both groups increased their self-reported physical activity levels from baseline, suggesting a measurement effect. The finding that the intervention group spent more time being active in their leisure time is of interest. Of the handful of previous smoking cessation trials that have measured or reported physical activity levels of the control group, findings have been mixed. Three studies [32-34] found a statistically significant effect on exercise levels in favor of the intervention group at the end of treatment, while three others found no differences [35-37]. Of the studies that reported an effect at the end of treatment, only one found that this effect was maintained at 1-year of follow-up [32]. As in our study, three trials reported increased levels of exercise in the control group from end of treatment to follow-up [33, 34, 38], which may have attenuated any effect of the intervention. Self-reported walking also increased to a much greater extent in the intervention group than the control group, which again was a target of the intervention.

Adherence to the intervention was important. Sensitivity analyses showed that participants who received at least $70 \%$ or more of the allocated intervention contacts were less likely to smoke at the end of the intervention and were $33 \%$ more likely to have quit compared to those in the control condition. Given there was no attention-control condition, it is unclear

Table 4 Change in physical activity outcomes estimated (repeated measures mixed model adjusted for baseline outcome, stratification factors, age, and strength of urge to smoke) at 24 weeks

\begin{tabular}{|c|c|c|c|c|c|c|}
\hline \multirow{2}{*}{$\begin{array}{l}\text { International physical activity questionnaire } \\
\text { domain (MET minutes/week) }\end{array}$} & \multirow{2}{*}{$\begin{array}{l}\text { Intervention } \\
\text { Estimate (SE) }\end{array}$} & \multirow{2}{*}{$\begin{array}{l}\text { Control } \\
\text { Estimate (SE) }\end{array}$} & \multicolumn{4}{|c|}{ Estimated effect size (Intervention-control) } \\
\hline & & & $\begin{array}{l}\text { Difference in } \\
\text { means (SE) }\end{array}$ & $95 \%$ lower $\mathrm{CI}$ & $95 \%$ higher CI & $P$-value \\
\hline Leisure-time physical activity & $526(69.2)$ & $307(66.1)$ & $219.1(84.8)$ & 52.7 & 385.6 & 0.01 \\
\hline Work domain & $580.9(313.8)$ & $538.1(301.2)$ & $42.8(384.9)$ & -712.8 & 798.4 & 0.91 \\
\hline Active transport domain & $164.6(52.9)$ & $101.9(50.7)$ & $62.7(65.0)$ & -64.9 & 190.3 & 0.34 \\
\hline Domestic and garden domain & $119.6(124.8)$ & $259.2(119.7)$ & $-139.6(153.6)$ & -441.1 & 162.0 & 0.36 \\
\hline Total walking & $364.5(153.2)$ & $207.9(126.5)$ & $156.6(160.8)$ & -159.2 & 472.3 & 0.33 \\
\hline Total moderate physical activity & $330.8(173.0)$ & $410.1(165.1)$ & $-79.3(211.6)$ & -494.8 & 336.2 & 0.71 \\
\hline Total vigorous physical activity & $871.4(225.2)$ & $725.0(215.6)$ & $146.4(277.2)$ & -397.8 & 690.6 & 0.60 \\
\hline
\end{tabular}


whether this effect is a result of the intervention content or the greater contact time received. This is an important finding and suggests that exercise counseling interventions may have an effect on smoking abstinence for those who choose to use them. In the present study, we did not assess participant's readiness to initiate exercise; however, identifying smokers who want to quit and screening for readiness to exercise may enhance adherence to an exercise intervention and thus maximize smoking cessation outcomes.

Strengths of this trial include its large sample size (it is the largest of its kind, excluding one Internet-based trial [36]); the public health approach and pragmatic nature of the trial, which incorporated existing and effective behavior change programs; the peer-reviewed design process; the use of reliable and valid measures; and the ethnically diverse sample, which differs from previous trials that have included predominantly white populations. Notwithstanding these strengths, and attempts to address methodological limitations associated with previous research, the present study findings are consistent with other randomized controlled trials conducted in this area [6]. The few trials that have been conducted, involving physical activity counseling, have yet to demonstrate an effective approach to increasing physical activity in a way that may impact on smoking cessation.

One of the proposed mechanisms for exercise helping people quit is mitigation of the negative effects of tobacco withdrawal symptoms. In the present trial, there was no intervention effect on tobacco withdrawal symptoms as measured with mood and physical symptoms scale, which in turn may contribute to the null effect of the intervention on quit rates. However, there was a small, but statistically significant difference in number of cigarettes smoked per day between groups, in favor of the intervention group. This latter finding may be important from a harm reduction perspective, which has been shown to be at least as effective as abrupt quitting on long term ( $>6$ months) quit rates [39]. Nevertheless, the clinical relevance of a difference of one cigarette smoked per day between groups is questionable.

Limitations of this trial include the slow recruitment rate and the subsequent reduction in sample size. The slow recruitment rate was identified early in the study, and various measures were implemented (including telephone assessments, employing additional staff for recruitment, and reducing participant burden by decreasing the number of measures) to improve this. While the final sample was below the initial target, based on the present findings, it is unlikely that a larger sample would have altered the conclusion. Second, there was relatively high and differential loss to follow-up, which is not uncommon in smoking cessation trials [32, 35, 40], but has implications when interpreting the findings. Using an intention-to-treat approach, participants who did not provide follow-up data were treated as smokers; thus, higher loss to follow-up potentially attenuated any intervention effect.
However, the sensitivity analysis using observed data also showed no intervention effect, suggesting the results are robust. Third, smoking cessation outcomes were self-reported, and as assessments were conducted predominantly via telephone, it was difficult to objectively verify abstinence. Verification of smoking status has been used to prevent overreporting of successful quit rates, and has particular relevance when differences in quit rates exist between groups. However, recent research has argued that biochemical verification of abstinence is outweighed by the burden (or cost) of obtaining such information [29]. It is important to note that cotinine verification was attempted, but the return rate was minimal. Fourth, it was not feasible to blind participants to their allocated study arm. Fifth, $30 \%$ of intervention group participants received less than half of the intervention calls, which given the effect of treatment adherence on smoking quit rates, significantly affected the success of the intervention. Lack of adherence to exercise is not uncommon among previous studies of exercise interventions for smoking cessation [32, 35, 36, $38,41]$. Finally, exercise was assessed by self-report, which is associated with recall bias.

\section{Future Research}

A key focus for future research is to test different forms of exercise prescription and to enhance adherence to exercise interventions. Previous research in the acute setting has shown that higher intensity exercise was associated with greater reductions in tobacco withdrawal symptoms and cravings even when quitters were using nicotine replacement therapy [42]. Thus, future studies might consider prescribing more brief bouts of exercise as a strategy for craving and withdrawal symptom relief, particularly in the first $2-3$ weeks of smoking cessation. With respect to the observed adherence effect, targeting an exercise intervention for those who are motivated to exercise or have greater readiness to change may have greater impact. If exercise works for some individuals and not others, perhaps interventions for smoking cessation should include exercise as one intervention component for those individuals who are motivated to do so.

Finally, where possible, physical activity should be measured objectively to avoid bias associated with self-report, and tobacco withdrawal symptoms should be assessed more proximal to the quit data (i.e., within the first week of smoking abstinence).

\section{Implications}

If the intervention had been shown to be effective we had planned to encourage the integration of two national services to increase national quit rates. Nevertheless, despite the lack of an effect of exercise on smoking cessation outcomes, physical activity is beneficial for a wide range of other health risks and 
outcomes such as depression, cardiovascular disease and type 2 diabetes. Therefore, it is important that physically inactive or insufficiently active people who smoke are referred to programs to increase activity levels. Moreover, people engaged in physical activity promotion programs such as Green Prescription who smoke should be actively referred to smoking cessation programs that provide evidence-based interventions, including pharmacotherapy and behavioral counseling from a health care professional. It is possible that increasing physical activity levels in those who smoke may not enhance quit rates but lead to a reduction in cigarette consumption, which may lead ultimately to cessation [43]. In the present study, we found that our intervention was associated with a reduction of only one cigarette per day; however as highlighted above, different exercise prescription may have a greater effect on cigarette consumption than observed here. While the Fit2Quit intervention content has potential, more focus is needed on increasing the uptake of the intervention.

\section{Conclusions}

A telephone-based exercise counseling intervention in addition to usual care did not augment abstinence rates at 24 weeks in adult smokers trying to quit. However, those who received at least $70 \%$ or more of the allocated intervention contacts were less likely to smoke at the end of 24 weeks. The exercise counseling intervention had a positive effect on leisure time activity and a negligible effect on cigarette consumption.

\begin{abstract}
Acknowledgments This was an investigator-initiated study funded by a grant from the Health Research Council of New Zealand (09/338R) and a small project grant from the Heart Foundation of New Zealand (1405). RM was supported by a Heart Foundation of New Zealand Fellowship. VR was supported by a University of Auckland Doctoral Scholarship. The funders played no role in the design, conduct or analysis of the study, nor in the interpretation and reporting of the study findings. The researchers were independent from the funders. All authors, external and internal, had full access to all of the data (including statistical reports and tables) in the study and can take responsibility for the integrity of the data and the accuracy of the data analysis.
\end{abstract}

Authors' Statement of Conflict of Interest and Adherence to Ethical Standards Ralph Maddison, Vaughan Roberts, Harry Prapavessis, Marewa Glover, Yannan Jiang, Paul Brown, William Leung, Sue Taylor, and Midi Tsai declare that they have no conflict of interest. Christopher Bullen has received support for accommodation while a speaker hosted by a manufacturer of smoking cessation drugs but has no other interests to declare. Hayden McRobbie has received honoraria for speaking at research symposia and received benefits in kind and travel support from and has provided consultancy to the manufacturers of smoking-cessation medications, specifically Pfizer, GSK, and J\&J. All procedures, including the informed consent process, were conducted in accordance with the ethical standards of the responsible committee on human experimentation (institutional and national) and with the Helsinki Declaration of 1975, as revised in 2000 .

\section{References}

1. US Department of Health \& Human Services. How tobacco smoke causes disease: the biology and behavioral basis for smokingattributable disease: a report of the surgeon general. Atlanta, GA: US Department of Health \& Human Services, Centers for Disease Control and Prevention, National Center for Chronic Disease Prevention and Health Promotion, Office on Smoking and Health; 2010.

2. World Health Organization. WHO report on the global tobacco epidemic, 2011: warning about the dangers of tobacco Geneva: World Health Organisation; 2011 [27/10/2012].

3. Cahill K, Stead LF, Lancaster T. Nicotine receptor partial agonists for smoking cessation. Cochrane Database of Systematic Reviews. 2012; 4, CD006103. doi:10.1002/14651858.CD006103.pub6.

4. Hughes JR, Stead LF, Lancaster T. Antidepressants for smoking cessation. Cochrane Database of Systematic Reviews. 2004(4): CD000031.pub2.

5. Stead LF, Perera R, Bullen C, Mant D, Lancaster T. Nicotine replacement therapy for smoking cessation. Cochrane Database of Systematic Reviews. 2008(1):CD000146. doi:10.1002/14651858. CD000146.pub3.

6. Ussher MH, Taylor A, Faulkner G. Exercise interventions for smoking cessation. Cochrane Database of Systematic Reviews (Online). 2012; 1, CD002295.

7. Benowitz NL. Neurobiology of nicotine addiction: implications for smoking cessation treatment. American Journal of Medicine. 2008; 121(4 Suppl 1): S3-10. doi:10.1016/j.amjmed.2008.01.015.

8. Taylor AH, Ussher MH, Faulkner G. The acute effects of exercise on cigarette cravings, withdrawal symptoms, affect and smoking behaviour: a systematic review. Addiction. 2007; 102(4): 534543.

9. Haasova M, Warren FC, Ussher M, et al. The acute effects of physical activity on cigarette cravings: systematic review and meta-analysis with individual participant data (IPD). Addiction. 2013; 108(1): 2637.

10. Roberts V, Maddison R, Simpson C, et al. The acute effects of exercise on cigarette cravings, withdrawal symptoms, affect, and smoking behaviour: systematic review update and meta-analysis. Psychopharmacology. 2012; 222(1): 1-15.

11. Marcus BH, Albrecht A, Niaura RS, et al. Usefulness of physical exercise for maintaining smoking cessation in women. American Journal of Cardiology. 1991; 68: 406-7.

12. Marcus BH, Albrecht AE, King TK, et al. The efficacy of exercise as an aid for smoking cessation in women: a randomized controlled trial. Archives of Internal Medicine. 1999; 159(11): 1229-34.

13. Martin JE, Calfas KJ, Patten CA, et al. Prospective evaluation of three smoking interventions in 205 recovering alcoholics: one-year results of Project SCRAP-Tobacco. Journal of Consulting \& Clinical Psychology. 1997; 65(1): 190-194.

14. The Quit Group. Quitline annual review 2010/2011. New Zealand; 2011. Available from: www.quit.org.nz/file/quit-ar2011-lo-.pdf.

15. McRobbie H. Treating smokers 'On Top' and 'Down Under'models of tobacco treatment from England and New Zealand. World Conference on Tobacco or Health; 13-15 July; Washington DC2006.

16. Elley R, Kerse N, Arroll B, et al. Effectiveness of counselling patients on physical activity in general practice: cluster randomised controlled trial. British Medical Journal. 2003; 326: 793-799.

17. Maddison R, Roberts V, Bullen C, et al. Design and conduct of a pragmatic randomized controlled trial to enhance smoking-cessation outcomes with exercise: The Fit2Quit study. Mental Health and Physical Activity. 2010; 3(2): 92-101.

18. Heatherton T, Kozlowski L, Frecker R, et al. The Fagerstrom Test for Nicotine Dependence: a revision of the Fagerstrom Tolerance Questionnaire. British Journal of Addiction. 1991; 86(9): 1119-1127. 
19. West R, Hajek P. Evaluation of the mood and physical symptoms scale (MPSS) to assess cigarette withdrawal. Psychopharmacology (Berl). 2004.

20. Craig CL, Marshall AJ, Sjöström M, et al. International Physical Activity Questionnaire: 12-country reliability and validity. Medicine \& Science in Sports \& Exercise. 2003; 35(8): 1381-1395.

21. Swinburn BA, Wlater LG, Arroll B, Tilyard MW, Russull DG. The green prescription study: A randomized controlled trial of written exercise advice provided by general practitioners. American Journal of Public Health. 1998; 88(2):288-91.

22. Nigg CR, Allegrante JP, Ory M. Theory-comparison and multiplebehavior research: common themes advancing health behavior research. Health Education Research. 2002; 17(5): 670-679.

23. U.S. Department of Health \& Human Services. Physical activity and health: a report of the surgeon general. Atlanta, GA: US Department of Health \& Human Services, Centres of Disease Control and Prevention, National Center for Chronic Disease Prevention and Health Promotion; 1996.

24. Bandura A. Social foundations of thought and action. New York: Prentice-Hall; 1986.

25. Sluijs EM, Knibbe JJ. Patient compliance with exercise: different theoretical approaches to short-term and long-term compliance. Patient Education and Counseling. 1991; 17: 191-204.

26. Baranowski T, Anderson C, Carmack C. Mediating variable framework in physical activity interventions. How are we doing? How might we do better? American Journal of Preventive Medicine. 1998; 15(4): 266-297.

27. Jeffery R. How can Health Behavior Theory be made more useful for intervention research? International Jounral of Behavioral Nutrition and Physical Activity. 2004; 1: 1-10.

28. Velicer WF, Prochaska JO, Rossi JS, et al. Assessing outcome in smoking cessation studies. Psychological Bulletin. 1992; 111(1): 23 41.

29. SRNT subcommittee on biochemical verification. Biochemical verification of tobacco use and cessation. Nicotine \& Tobacco Research. 2002; 4(2): 149-159.

30. West R, Hajek P, Stead L, et al. Outcome criteria in smoking cessation trials: proposal for a common standard. Addiction. 2005; 100(3): 299-303.

31. Bauman A, Ainsworth BE, Bull F, et al. Progress and pitfalls in the use of the International Physical Activity Questionnaire (IPAQ) for adult physical activity surveillance. Jounral of Physical Activity and Health. 2009; 6(Suppl 1): S5-S8.
32. Bize R, Willi C, Chiolero A, et al. Participation in a population-based physical activity programme as an aid for smoking cessation: a randomised trial. Tobacco Control: An International Journal. 2010; 19(6): 488-494.

33. Ciccolo JT, Dunsiger SI, Williams DM, et al. Resistance training as an aid to standard smoking cessation treatment: a pilot study. Nicotine and Tobacco Research. 2011; 13(8): 756-760.

34. Vickers KS, Patten CA, Lewis BA, et al. Feasibility of an exercise counseling intervention for depressed women smokers. Nicotine \& Tobacco Research. 2009; 11(8): 985-995.

35. Kinnunen T, Leeman RF, Korhonen T, et al. Exercise as an adjunct to nicotine gum in treating tobacco dependence among women. Nicotine and Tobacco Research. 2008; 10(4): 689-703.

36. McKay H, Danaher BG, Seeley JR, et al. Comparing two web-based smoking cessation programs: randomized controlled trial. Journal of Medical Internet Research. 2008; 10(5): 68-81.

37. Ussher M, West R, McEwen A, et al. Efficacy of exercise counselling as an aid for smoking cessation: a randomized controlled trial. Addiction. 2003; 98(4): 523-532.

38. Ussher M, West R, McEwen A, et al. Randomized controlled trial of physical activity counseling as an aid to smoking cessation: 12 month follow-up. Addictive Behaviors. 2007; 32(12): 3060-3064.

39. Lindson N, Aveyard P, Hughes JR. Reduction versus abrupt cessation in smokers who want to quit. Cochrane Database of Systematic Reviews. 2010; 3, CD008033. doi:10.1002/14651858.CD008033. pub2.

40. Walker N, Howe C, Bullen C, et al. The combined effect of very low nicotine content cigarettes, used as an adjunct to usual Quitline care (nicotine replacement therapy and behavioural support), on smoking cessation: a randomized controlled trial. Addiction. 2012:1857-1867. doi:10.1111/j.1360-0443.2012.03906.x.

41. Marcus B, Lewis BA, Hogan J, et al. The efficacy of moderateintensity exercise intervention as an aid for smoking cessation in women: a randomized controlled trial. Nicotine \& Tobacco Research. 2005; 7(6): 871.

42. Harper T, Fitzgeorge L, Tritter A, Prapavessis H. Acute exercise effects on craving and withdrawal symptoms among women attempting to quit smoking using nicotine replacement therapy. Journal of Smoking Cessation. 2012:Available on CJO. doi:10. 1017/jsc.2012.15.

43. Walker N, Bullen C, McRobbie H. Reduced-nicotine content cigarettes: is there potential to aid smoking cessation? Nicotine and Tobacco Research. 2009; 11(11): 1274-1279. doi:10.093/ntr/ntp147. 